\title{
Association Between Thyroid Hormones, Thyroid Antibodies, and Cardiometabolic Factors in Non-Obese Individuals With Normal Thyroid Function
}

\author{
Jia Liu, Yan Duan, Jing Fu and Guang Wang* \\ Department of Endocrinology, Beijing Chao-yang Hospital, Capital Medical University, Beijing, China
}

Background: Hypothyroidism is an important risk factor for cardiovascular diseases, and autoimmune thyroiditis (AIT) is the leading cause of hypothyroidism. Recent studies showed that even AIT patients with euthyroidism still had an increased number of early atherosclerotic lesions. However, the precise mechanism is not yet known. This study aimed to investigate the association of thyroid function, thyroid autoimmunity, and cardiometabolic risk factors in non-obese AIT patients with euthyroidism.

OPEN ACCESS

Edited by:

Yuji Nagayama,

Nagasaki University, Japan

Reviewed by:

Misa Imaizumi,

Radiation Effects Research

Foundation, Japan

Onyebuchi Okosieme,

Cwm Taf University Health Board,

United Kingdom

*Correspondence:

Guang Wang

drwg6688@126.com

Specialty section:

This article was submitted

to Thyroid Endocrinology,

a section of the journal

Frontiers in Endocrinology

Received: 27 January 2018

Accepted: 13 March 2018

Published: 05 April 2018

Citation:

Liu J, Duan Y, Fu J and Wang G (2018) Association Between Thyroid Hormones, Thyroid Antibodies, and Cardiometabolic Factors in

Non-Obese Individuals With Normal Thyroid Function.

Front. Endocrinol. 9:130.

doi: 10.3389/fendo.2018.00130
Methods: A total of 5,608 non-obese individuals including 1,402 AIT patient and 4,206 sex-, age-, and body mass index (BMI)-matched healthy controls were recruited.

Results: The AIT patients had significantly lower free T3 and free T4 levels, and higher TSH, antithyroid peroxidase antibodies (TPOAb) and TgAb levels. The elevated levels of high sensitivity $\mathrm{C}$ reactive protein (hsCRP) and homeostasis model assessment of insulin resistance (HOMA-IR) were observed in the AIT patients than the controls [hsCRP: 0.65 (0.27-1.33) vs. 0.20 (0.03-0.74) mg/L; HOMA-IR: $2.78 \pm 1.60$ vs. $2.33 \pm 1.49$; all $P<0.05]$. Thyroid function was not associated with metabolic parameters and inflammatory makers, while the TPOAb titer was positively associated with the HOMA-IR and hsCRP levels after adjustment for confounding factors (all $P<0.05$ ). Multivariate regression analysis demonstrated that the TPOAb level was an independent influencing factor for the HOMA-IR and hsCRP levels (HOMA-IR: $\beta=0.058, P<0.05$; hsCRP: $\beta=0.108, P<0.05)$.

Conclusion: The TPOAb level is associated with HOMA-IR and hsCRP levels independently of thyroid function in non-obese individuals. Mild deviation of thyroid function within the normal range, chronic inflammation, and insulin resistance may be the links between AIT and atherosclerosis in the non-obese population.

Keywords: autoimmune thyroiditis, cardiovascular disease, atherosclerosis, chronic inflammation, insulin resistance

\section{INTRODUCTION}

Autoimmune thyroiditis (AIT) is a common endocrine disease characterized by diffuse thyroid enlargement and lymphocytic infiltration $(1,2)$. AIT manifests in patients through different states of thyroid function, such as euthyroidism or subclinical or overt hypothyroidism (1). Subclinical or overt hypothyroidism is an important risk factor for cardiovascular diseases (3). Many 
studies, including our previous studies, found that hypothyroidism causes insulin resistance, dyslipidemia, and chronic inflammation and further contributes to the development of atherosclerosis $(4,5)$. Notably, evidence from recent studies has shown that even AIT patients in euthyroid still have increased number of early atherosclerotic lesions $(6,7)$. However, the precise mechanism has not yet been revealed. Previous studies have shown that euthyroid AIT patients have relatively higher TSH levels than controls, and the TSH levels were positively associated with insulin resistance (8-10). Moreover, elevated total cholesterol (TC) levels were also present in euthyroid AIT patients (11). However, these results are inconsistent, and most of this evidence comes from an obese population. Obesity is a primary risk factor for insulin resistance and a chronic inflammatory state, and also affects thyroid function (12-15). Therefore, obesity might influence the reliability of results. This study aimed to investigate the association of thyroid function, thyroid autoimmunity, and cardiometabolic risk factors in non-obese AIT patients with euthyroidism.

\section{MATERIALS AND METHODS}

\section{Study Population}

A total of 1,402 non-obese AIT patients were recruited from Endocrinology Department of Beijing Chao-Yang Hospital affiliated with Capital Medical University from June 2015 through July 2016. Every AIT patient was matched by three sex-, age-, and body mass index (BMI)-matched healthy individuals who were recruited from the Physical Examination Center of Beijing Chao-Yang Hospital Affiliated to Capital Medical University. Finally, 4,206 sex-, age-, and BMI-matched healthy individuals were included as the control group. Non-obese individuals were diagnosed if their BMI $<30 \mathrm{~kg} / \mathrm{m}^{2}$. Oral glucose tolerance tests, thyroid function tests, and thyroid ultrasonography were performed at screening. Individuals with thyroid dysfunction, hypertension, diabetes, coronary artery disease, liver or renal function impairment, infectious disease, systemic inflammatory disease, or cancer were excluded. No subject took lipid-lowering agents, levothyroxine, or antithyroid drugs. Participants who were pregnant, possibly pregnant, or ingesting agents known to influence thyroid function were also excluded. Subjects with an abnormal free T4 (FT4) concentration (reference range: 12.00-22.00 pmol/L) or TSH concentration (reference range: 0.51-4.94 IU/mL) were excluded. AIT was diagnosed by elevated antithyroid peroxidase antibodies (TPOAb) (reference range: 0.00-60.00 U/mL) and/or antithyroglobulin antibodies (TgAb) (reference range: $0.00-60.00 \mathrm{U} / \mathrm{mL}$ ) and typical hypoechogenicity of the thyroid in a thyroid ultrasound (6). All enrolled subjects provided written informed consent, and the protocol of this study was approved by the Ethics Committee of the Beijing ChaoYang Hospital, Capital Medical University.

\section{Clinical and Biochemical Measurements}

A standard questionnaire was used to collect information about each patient's health status and medications. Height and weight were measured to the nearest $0.1 \mathrm{~cm}$ and $0.1 \mathrm{~kg}$ by the same trained group, respectively. Venous blood samples were obtained after overnight fasting. Blood samples of all participants were stored at $-80^{\circ} \mathrm{C}$. High-density lipoprotein cholesterol (HDL-C), low-density lipoprotein cholesterol (LDL-C), triglyceride (TG), and TC levels were measured by colorimetric enzymatic assays using an autoanalyzer (Hitachi 7170). Serum TC was measured by an enzymatic cholesterol oxidase reaction, TG by a glycerol lipase oxidase reaction, and HDL-C and LDL-C were measured by a direct assay. Fasting blood glucose (FBG) was measured by the glucose oxidase method, and fasting insulin (FINS) by the chemiluminescence method. High sensitivity $\mathrm{C}$ reactive protein (hsCRP) was measured with an immunonephelometric assay. Free T3 (FT3), FT4, and TSH were determined by electrochemiluminescence immunoassay using an Abbott Architect i2000 (Abbott Diagnostics, Abbott Park, IL, USA), and the intra- and interassay coefficient of variation was below $10 \%$ for FT3, FT4, and TSH. The serum concentrations of $\mathrm{TgAb}$ and TPOAb were detected by chemiluminescent immunoassay. For both TgAb and TPOAb, the intra- and interassay coefficient of variation was below $10 \%$. BMI was calculated as the weight in kilograms divided by the height in square meters. Homeostasis model assessment of insulin resistance (HOMA-IR) was performed to evaluate insulin resistance according to the following formula: HOMA-IR $=[$ FINS $(\mu \mathrm{IU} /$ $\left.\mathrm{mL})^{\star} \mathrm{FBG}(\mathrm{mmol} / \mathrm{L}) / 22.5\right]$ (16). Thyroid ultrasound was assessed by a well-trained ultrasound physician.

\section{Statistical Methods}

Normally distributed variables were expressed as mean $\pm \mathrm{SD}$, while variables with a skewed distribution, including TG, FINS, $\mathrm{TSH}, \mathrm{TPOAb}$, and TgAb, were given as median and upper and lower quartiles. Variables that were not normally distributed were log-transformed before analysis. The differences between two groups were analyzed by independent Student's $t$-test or the Mann-Whitney $U$ test. The proportions were analyzed using chi-square tests. The differences between groups were analyzed by ANOVA test. We also performed Pearson and Spearman correlation analyses. Bonferroni correction was further made for multiple correlations. Multivariate stepwise regression analysis was used to evaluate the relationship between parameters. All statistical analyses were performed with SPSS 17.0 (SPSS, Inc., Chicago, IL, USA), and the results were considered statistically significant with two-tailed analyses, $P<0.05$.

\section{RESULTS}

\section{Clinical Characteristics of the Control and AIT Groups}

The clinical characteristics of the participants are summarized in Table 1. The control and AIT groups did not significantly differ in age, gender, BMI, TC, LDL-C, HDL-C, and TG. The levels of FBG and FINS were also statistically the same between the two groups. AIT patients had significantly lower FT3 and FT4 levels, and higher TSH, TPOAb, and TgAb levels, compared with the controls [FT3: $4.94 \pm 0.58$ vs. $5.15 \pm 0.58 \mathrm{pmol} / \mathrm{L}$; FT4: $15.76 \pm 2.00$ vs. $16.17 \pm 1.92 \mathrm{pmol} / \mathrm{L}$; TSH: $2.11(1.47-2.98)$ vs. $1.62(1.20-2.20)$ $\mathrm{mIU} / \mathrm{mL}$; TPOAb: $135.75(46.70-1,248.70)$ vs. $29.10(0-38.00)$ IU/mL; TgAb: 131.90 (61.30-241.00) vs. 15.60 (0-22.80) IU/mL; all $P<0.01$; Table 1]. The increased levels of hsCRP and HOMA-IR 
were observed in the AIT patients compared with the controls [hsCRP: 0.65 (0.27-1.33) vs. 0.20 (0.03-0.74) mg/L; HOMA-IR: $2.78 \pm 1.60$ vs. $2.33 \pm 1.49$; all $P<0.05$; Table 1$]$.

\section{Correlation Between Thyroid Function, Thyroid Antibodies, and Clinical Parameters}

Correlation between thyroid function, thyroid antibodies, and clinical parameters is presented in Table 2. FT3 was positively

TABLE 1 | Clinical characteristics of the control and AIT groups.

\begin{tabular}{|c|c|c|c|}
\hline Parameters & $\begin{array}{l}\text { Control group } \\
(n=4,206)\end{array}$ & $\begin{array}{l}\text { AlT group } \\
(n=1,402)\end{array}$ & $P$ \\
\hline Age, years & $45.50 \pm 12.56$ & $45.97 \pm 12.08$ & 0.128 \\
\hline Gender, F/M & $3,098 / 1,108$ & 1,036/366 & 0.334 \\
\hline $\mathrm{BMI}, \mathrm{kg} / \mathrm{m}^{2}$ & $23.98 \pm 2.70$ & $24.01 \pm 3.18$ & 0.740 \\
\hline $\mathrm{TC}, \mathrm{mmol} / \mathrm{L}$ & $5.01 \pm 0.92$ & $5.02 \pm 0.97$ & 0.739 \\
\hline LDL-C, mmol/L & $2.73 \pm 0.73$ & $2.72 \pm 0.74$ & 0.538 \\
\hline HDL-C, mmol/L & $1.31 \pm 0.32$ & $1.33 \pm 0.38$ & 0.063 \\
\hline $\mathrm{TG}, \mathrm{mmol} / \mathrm{L}$ & $1.13(0.81-1.66)$ & $1.10(0.79-1.58)$ & 0.054 \\
\hline $\mathrm{FBG}, \mathrm{mmol} / \mathrm{L}$ & $5.41 \pm 0.50$ & $5.39 \pm 0.48$ & 0.075 \\
\hline FINS, $\mu \mathrm{IU} / \mathrm{mL}$ & $9.61(6.72-13.35)$ & $9.91(7.33-13.82)$ & 0.124 \\
\hline HOMA-IR & $2.33 \pm 1.49$ & $2.78 \pm 1.60$ & 0.021 \\
\hline hsCRP, mg/L & $0.20(0.03-0.74)$ & $0.65(0.27-1.33)$ & 0.027 \\
\hline FT3, pmol/L & $5.15 \pm 0.58$ & $4.94 \pm 0.58$ & 0.000 \\
\hline FT4, pmol/L & $16.17 \pm 1.92$ & $15.76 \pm 2.00$ & 0.000 \\
\hline TSH, mlU/L & $1.62(1.20-2.20)$ & $2.11(1.47-2.98)$ & 0.000 \\
\hline TPOAb, IU/mL & $29.10(0-38.00)$ & $135.75(46.70-1,248.70)$ & 0.000 \\
\hline TgAb, IU/mL & $15.60(0-22.80)$ & $131.90(61.30-241.00)$ & 0.000 \\
\hline
\end{tabular}

Data are means $\pm S D$ unless indicated otherwise.

TG, FINS, TSH, TPOAb, and TgAb are shown as median and upper and lower quartiles.

$B M I$, body mass index; TC, total cholesterol; $L D L-C$, low-density lipoprotein cholesterol; HDL-C, high-density lipoprotein cholesterol; TG, triglyceride; FBG, fasting blood glucose; FINS, fasting insulin; HOMA-IR, homeostasis model assessment of insulin resistance; hSCRP, high sensitivity C reactive protein; FT3, free T3; FT4, free T4; TPOAb, antithyroid peroxidase antibodies; TgAb, antithyroglobulin antibodies; AIT, autoimmune thyroiditis. associated with BMI, LDL-C, TG, FBG, FINS, and HOMA-IR and negatively associated with age, TC, and HDL-C (Table 2). FT4 levels were positively associated with LDL-C, TG, and FBG, and negatively associated with age, BMI, HDL-C, and HOMA-IR (Table 2). We also found the positive association between TSH and TC (Table 2). However, these associations lost their significance after the adjustment using Bonferroni correction.

The TPOAb level was positively associated with TSH, TC, TG, FINS, HOMA-IR, and hsCRP, and negatively associated with FT3 and FT4 (Table 2). The TgAb level was positively associated with TSH and negatively associated with BMI, FT3, and FT4 (Table 2). The levels of TPOAb and TgAb were positively associated with $\mathrm{TSH}$, and negatively associated with FT3 even after Bonferroni correction. Furthermore, after Bonferroni correction, TPOAb still showed a significant association with HOMA-IR and hsCRP (all $P<0.05$ ), but the association between TgAb and BMI had disappeared.

\section{Clinical Characteristics of AIT Groups With Different TPOAb Titers}

In the previous studies, the high TPOAb titer was frequently defined when the TPOAb titer was greater than $1,000 \mathrm{IU} / \mathrm{mL}$ (17). Therefore, we further divided all AIT patients into two subgroups according to whether their TPOAb titers were greater than 1,000 IU/mL: the low TPOAb AIT group (AIT patients with TPOAb $<1,000 \mathrm{IU} / \mathrm{mL}$ ) and high TPOAb AIT group (AIT patients with $\mathrm{TPOAb} \geq 1,000 \mathrm{IU} / \mathrm{mL}$ ). The clinical characteristics of the non-obese and euthyroid individuals with different TPOAb titers are summarized in Table 3. There was no significant difference in age, gender, BMI, TC, LDL-C, HDL-C, TG, FBG, and FINS among the control group, the low TPOAb AIT group, and the high TPOAb AIT group. The patients in both the low TPOAb AIT group and the high TPOAb AIT group have decreased FT3, FT4 levels, and increased HOMA-IR, TSH, TPOAb, and TgAb levels than the healthy controls (Table 3). The high TPOAb AIT group has higher hsCRP levels than the control group [0.98

TABLE 2 | Correlation between thyroid function, thyroid antibodies, and metabolic parameters.

\begin{tabular}{|c|c|c|c|c|c|c|c|c|c|c|}
\hline & \multicolumn{2}{|c|}{ FT3 } & \multicolumn{2}{|c|}{ FT4 } & \multicolumn{2}{|c|}{ TSH } & \multicolumn{2}{|c|}{ TPOAb } & \multicolumn{2}{|c|}{ TgAb } \\
\hline Age & -0.135 & 0.000 & -0.014 & 0.000 & -0.006 & 0.660 & 0.019 & 0.164 & 0.004 & 0.775 \\
\hline TC & -0.052 & 0.000 & -0.004 & 0.749 & 0.029 & 0.035 & 0.042 & 0.002 & 0.015 & 0.276 \\
\hline LDL-C & 0.032 & 0.023 & 0.037 & 0.008 & -0.009 & 0.524 & 0.015 & 0.279 & -0.008 & 0.574 \\
\hline HDL-C & -0.035 & 0.025 & -0.028 & 0.043 & 0.016 & 0.234 & -0.008 & 0.547 & 0.012 & 0.316 \\
\hline FINS & 0.184 & 0.000 & -0.005 & 0.328 & 0.032 & 0.114 & 0.063 & 0.001 & 0.023 & 0.266 \\
\hline HOMA-IR & 0.188 & 0.000 & -0.052 & 0.011 & 0.020 & 0.332 & 0.065 & 0.001 & 0.027 & 0.197 \\
\hline hsCRP & 0.056 & 0.657 & -0.035 & 0.779 & -0.134 & 0.286 & 0.119 & 0.001 & 0.063 & 0.218 \\
\hline FT3 & - & - & - & - & - & - & -0.065 & 0.000 & -0.133 & 0.000 \\
\hline $\mathrm{FT} 4$ & - & - & - & - & - & - & -0.039 & 0.004 & -0.036 & 0.008 \\
\hline TSH & - & - & - & - & - & - & 0.162 & 0.000 & 0.178 & 0.000 \\
\hline
\end{tabular}

BMI, body mass index; TC, total cholesterol; LDL-C, low-density lipoprotein cholesterol; HDL-C, high-density lipoprotein cholesterol; TG, triglyceride; FBG, fasting blood glucose; FINS, fasting insulin; HOMA-IR, homeostasis model assessment of insulin resistance; hSCRP, high sensitivity C reactive protein; FT3, free T3; FT4, free T4; TPOAb, antithyroid peroxidase antibodies; TgAb, antithyroglobulin antibodies. 
TABLE 3 | Clinical characteristics of non-obese individuals with different TPOAb titers.

\begin{tabular}{|c|c|c|c|}
\hline & Control group ( $n=4,206)$ & Low TPOAb AIT group ( $n=1,031)$ & High TPOAb AIT group $(n=371)$ \\
\hline Age, years & $45.50 \pm 12.56$ & $46.21 \pm 12.13$ & $45.33 \pm 11.95$ \\
\hline Gender, F/M & $3,098 / 1,108$ & $751 / 280$ & $285 / 86$ \\
\hline $\mathrm{BMl}, \mathrm{kg} / \mathrm{m}^{2}$ & $23.98 \pm 2.70$ & $24.03 \pm 3.17$ & $23.98 \pm 3.23$ \\
\hline $\mathrm{TC}, \mathrm{mmol} / \mathrm{L}$ & $5.01 \pm 0.92$ & $5.02 \pm 0.88$ & $4.99 \pm 1.00$ \\
\hline LDL-C, mmol/L & $2.73 \pm 0.73$ & $2.74 \pm 0.86$ & $2.71 \pm 0.68$ \\
\hline HDL-C, mmol/L & $1.31 \pm 0.32$ & $1.34 \pm 0.32$ & $1.32 \pm 0.35$ \\
\hline $\mathrm{TG}, \mathrm{mmol} / \mathrm{L}$ & $1.13(0.81-1.66)$ & $1.11(0.79-1.57)$ & $1.09(0.78-1.58)$ \\
\hline FBG, mmol/L & $5.41 \pm 0.50$ & $5.38 \pm 0.48$ & $5.40 \pm 0.47$ \\
\hline FINS, $\mu \mathrm{IU} / \mathrm{mL}$ & 9.67 (6.74-13.36) & $9.74(7.19-13.85)$ & $10.55(7.72-13.81)$ \\
\hline HOMA-IR & $2.33 \pm 1.49$ & $2.74 \pm 1.60^{\star}$ & $2.90 \pm 1.58^{\star \S}$ \\
\hline hsCRP, mg/L & $0.20(0.03-0.74)$ & $0.41(0.06-0.65)$ & $0.98(0.77-1.36)^{\star \S}$ \\
\hline $\mathrm{FT} 3, \mathrm{pg} / \mathrm{mL}$ & $5.15 \pm 0.58$ & $4.95 \pm 0.61^{*}$ & $4.91 \pm 0.50^{*}$ \\
\hline $\mathrm{FT} 4, \mathrm{ng} / \mathrm{dL}$ & $16.17 \pm 1.92$ & $15.88 \pm 2.00^{\star}$ & $15.42 \pm 1.95^{\star \S}$ \\
\hline $\mathrm{TSH}, \mu \mathrm{IU} / \mathrm{mL}$ & $1.62(1.20-2.20)$ & $2.00(1.42-2.82)^{\star}$ & $2.46(1.69-3.32)^{\star \S}$ \\
\hline TPOAb, IU/ml & $29.10(0-38.00)$ & $67.55(35.70-100.03)^{\star \star}$ & $1,300.00(1,300.00-1,300.00)^{\star *, \S \S}$ \\
\hline TgAb, IU/ml & $15.60(0-22.80)$ & $122.30(55.30-231.30)^{\star \star}$ & $157.45(76.55-291.65)^{\star \star}$ \\
\hline
\end{tabular}

Data are means $\pm S D$ unless indicated otherwise.

TG, FINS, TSH, TPOAb, and TgAb are shown as median and upper and lower quartiles.

BMI, body mass index; TC, total cholesterol; LDL-C, low-density lipoprotein cholesterol; HDL-C, high-density lipoprotein cholesterol; TG, triglyceride; FBG, fasting blood glucose;

FINS, fasting insulin; HOMA-IR, homeostasis model assessment of insulin resistance; hsCRP, high sensitivity C reactive protein; FT3, free T3; FT4, free T4; TPOAb, antithyroid

peroxidase antibodies; TgAb, antithyroglobulin antibodies; AlT, autoimmune thyroiditis.

The low TPOAb AIT group (AlT patients with TPOAb <1,000 IU/mL) and high TPOAb AIT group (AIT patients with TPOAb $\geq 1,000 \mathrm{IU} / \mathrm{mL}$ ).

*Significantly different at $P<0.05$ vs. the control group.

${ }^{*}$ Significantly different at $P<0.01$ vs. the control group.

sSignificantly different at $P<0.05$ vs. the low TPOAb AlT group.

\$Significantly different at $P<0.01$ vs. the low TPOAb AIT group.

(0.77-1.36) vs. 0.20 (0.03-0.74), $P<0.05$; Table 3]. The levels of HOMA-IR, hsCRP, FT4, TSH, and TPOAb were significantly increased in the high TPOAb AIT group than the low TPOAb AIT group (Table 3).

\section{Multivariate Stepwise Regression Analysis}

To further evaluate the relationship between TPOAb and HOMAIR and hsCRP levels, we performed a multivariate regression analysis, including age, gender, BMI, TG, FT3, FT4, TSH, TPOAb, and TgAb. We found that the TPOAb level was an independent influencing factor for HOMA-IR and hsCRP levels (HOMA-IR: $\beta=0.058, P<0.05$; hsCRP: $\beta=0.108, P<0.05$ ).

\section{DISCUSSION}

This study showed that in a non-obese population with euthyroidism, the patients with AIT had significantly lower FT3 and FT4 levels, and higher TSH, TPOAb, and TgAb levels than the healthy controls. The elevated levels of hsCRP and HOMA-IR were also observed in the AIT patients as compared with the control group. Thyroid function was not associated with metabolic parameters and inflammatory makers, while the TPOAb titer was positively associated with the HOMA-IR and hsCRP levels after adjustment for confounding factors. Multivariate regression analysis demonstrated that the TPOAb level was an independent influencing factor for HOMA-IR and hsCRP levels.

Many epidemiological studies have shown that a certain percentage of AIT patients manifested as euthyroidism (1). Increasing evidence indicated that even AIT patients with euthyroidism still had an increased number of early atherosclerotic lesions $(6,7)$. This study showed that, although their thyroid function was within the normal range, the non-obese AIT patients had relatively lower FT3 and FT4 levels, and higher TSH than the controls. A recent cohort study from the euthyroid population showed that a low baseline FT4 level was associated with a high risk of the progression of coronary artery calcification over 4 years (18). An in vitro study also found that T3 inhibits the calcification and phenotype transformation of vascular smooth muscle cells (19). In vivo, recombinant TSH reduces endothelium-dependent vasodilation in patients with differentiated thyroid carcinoma (20). Furthermore, elevated TSH induces endothelial dysfunction in human umbilical vein endothelial cells by inhibiting eNOS expression (21). In euthyroid, patients who underwent coronary angiography, variation of thyroid function within normal range was associated with the severity of coronary atherosclerosis (22). Therefore, relatively lower FT3 and FT4 levels, and higher TSH levels might directly lead to the progression of atherosclerosis.

In this study, non-obese AIT patients with euthyroidism showed higher hsCRP levels than the controls, which suggested a chronic inflammatory state in AIT patients. This result was in line with previous studies. AIT patients had increased hsCRP, IL-6, and IL-1 $\beta$ levels $(7,23,24)$. Elevated levels of sICAM-1 and sVCAM-1 were also observed in euthyroid AIT patients compared with the controls (25). Our previous clinical and animal studies showed that the chronic inflammation cause endothelial dysfunction and atherosclerosis $(26,27)$. Thus, a chronic inflammatory status might be associated with the pathogenesis of atherosclerosis in euthyroid AIT patients.

Autoimmune thyroiditis is characterized by diffuse apoptosis of thyroid follicular cells induced by abnormal cellular and humoral immunity $(1,2)$. TPOAb, an important antibody for humoral immunity, is mainly produced by lymphocytes in the 
thyroid $(28,29)$. AIT patients with increased TPOAb levels have a relatively higher risk for developing hypothyroidism (30). A significant correlation between the TPOAb levels and the number of lymphocytes in the thyroid gland was demonstrated (28). Our study showed that the TPOAb level was positively associated with hsCRP. Previous studies have demonstrated that TPOAb induces cellular cytotoxicity and the complement system and further deteriorated the inflammatory status $(2,28)$. On the other hand, increased inflammatory factors directly damage thyroid cells, induce more lymphocyte infiltration, and further stimulate the production of $\operatorname{TPOAb}(2,29)$. Carotid intima media thickness (CIMT) is an indicator of early atherosclerosis (31). Several studies have found the positive association between CIMT and thyroid autoantibodies in euthyroid AIT patients (6). Thus, the chronic inflammation may be the link between thyroid autoimmunity and atherosclerosis in AIT patients.

It is well known that hypothyroidism is an important risk factor for metabolic disorders (32). Recently, increased interest has focused on the relationship between thyroid function and metabolic disorders in euthyroid population. This study showed that euthyroid, non-obese AIT patients had higher HOMA-IR levels when compared with the healthy controls. Previous studies have indicated that insulin is critical for a healthy endothelium by inducing the phosphorylation of PI3-K/Akt (33). The activation of the PI3-K/Akt pathway further increased the expression and phosphorylation of eNOS and promoted the production of NO (34). Thus, insulin resistance might contribute to endothelial dysfunction by impairing the PI3-K/Akt pathway and reducing the expression of eNOS and bioavailability of NO. Previous studies have shown that FT4 has an inverse association with HOMA-IR in a euthyroid population with obesity $(8,35)$. In addition, some studies have found a significant positive correlation between FT3 and hyperinsulinemia $(36,37)$. An association between TSH and HOMA-IR was also found in some other studies (35). However, most of this evidence came from studies of an obese population or small-scale studies. Obesity not only affects thyroid function but is also a primary risk factor for insulin resistance (12-15). Therefore, obesity might influence the reliability of results. This study showed that FT3 was positively associated with HOMA-IR, while FT4 levels were negatively associated with HOMA-IR. However, these associations lost their significance after adjustment for confounding factors. Consistently, a recent study found an association between thyroid function and metabolic parameters in obese patients with polycystic ovary syndrome (PCOS), while this association disappeared in non-obese PCOS patients (38). These results

\section{REFERENCES}

1. Antonelli A, Ferrari SM, Corrado A, Di Domenicantonio A, Fallahi P. Autoimmune thyroid disorders. Autoimmun Rev (2015) 14:174-80. doi:10.1016/ j.autrev.2014.10.016

2. Mikos H, Mikos M, Obara-Moszynska M, Niedziela M. The role of the immune system and cytokines involved in the pathogenesis of autoimmune thyroid disease (AITD). Endokrynol Pol (2014) 65:150-5. doi:10.5603/EP. 2014.0021

3. Selmer C, Olesen JB, Hansen ML, von Kappelgaard LM, Madsen JC, Hansen PR, et al. Subclinical and overt thyroid dysfunction and risk of all-cause mortality might suggest that the link between thyroid function and metabolic features is modulated by body weight. Although thyroid function had no relationship with insulin resistance, TPOAb levels were significantly associated with HOMA-IR levels. As mentioned earlier, TPOAb is associated with chronic inflammation. Thus, increased inflammatory status might contribute to increased insulin resistance in non-obese AIT patients with euthyroidism.

\section{CONCLUSION}

In a non-obese population with euthyroidism, the AIT patients had lower FT3 and FT4 levels, and higher levels of TSH, hsCRP, and HOMA-IR. The TPOAb level is associated with HOMA-IR and hsCRP levels independently of thyroid function. Mild deviation of thyroid function within the normal range, chronic inflammation, and insulin resistance may be the links between AIT and atherosclerosis in the non-obese population. More attention should be focused on AIT patients for cardiovascular disease even if they are euthyroid. And further prospective or intervention researches are needed to evaluate whether levothyroxine treatment will bring benefits in reducing atherosclerosis risk for the AIT patients with euthyroidism, especially in patients with high TPOAb titers.

\section{ETHICS STATEMENT}

All enrolled subjects provided written informed consent, and the protocol of this study was approved by the Ethics Committee of the Beijing Chao-Yang Hospital, Capital Medical University.

\section{AUTHOR CONTRIBUTIONS}

JL and GW conceived and designed the study and wrote the paper; JL, JF, YD, and GW performed the study; JL and JF analyzed the data.

\section{FUNDING}

This work was supported by grants from the Chinese National Natural Science Foundation (No. 81600657) and Capital Funds for Health Improvement and Research (No. 2018-4-2034) to JL, and the Chinese National Natural Science Foundation (No. 81770792) and Capital Clinical Research Foundation of Beijing Municipal Commission of Science and Technology (No. Z161100000516069) to GW.

and cardiovascular events: a large population study. J Clin Endocrinol Metab (2014) 99:2372-82. doi:10.1210/jc.2013-4184

4. Yang N, Yao Z, Miao L, Liu J, Gao X, Fan H, et al. Novel clinical evidence of an association between homocysteine and insulin resistance in patients with hypothyroidism or subclinical hypothyroidism. PLoS One (2015) 10:e0125922. doi:10.1371/journal.pone.0125922

5. Lu M, Yang CB, Gao L, Zhao JJ. Mechanism of subclinical hypothyroidism accelerating endothelial dysfunction (review). Exp Ther Med (2015) 9:3-10. doi:10.3892/etm.2014.2037

6. Topaloglu O, Gokay F, Kucukler K, Burnik FS, Mete T, Yavuz HC, et al. Is autoimmune thyroiditis a risk factor for early atherosclerosis in premenopausal 
women even if in euthyroid status? Endocrine (2013) 44:145-51. doi:10.1007/ s12020-012-9842-5

7. Isguven P, Gunduz Y, Kilic M. Effects of thyroid autoimmunity on early atherosclerosis in euthyroid girls with Hashimoto's thyroiditis. J Clin Res Pediatr Endocrinol (2016) 8:150-6. doi:10.4274/jcrpe.2145

8. Temizkan S, Balaforlou B, Ozderya A, Avci M, Aydin K, Karaman S, et al. Effects of thyrotrophin, thyroid hormones and thyroid antibodies on metabolic parameters in a euthyroid population with obesity. Clin Endocrinol (Oxf) (2016) 85:616-23. doi:10.1111/cen.13095

9. Amouzegar A, Kazemian E, Gharibzadeh S, Mehran L, Tohidi M, Azizi F. Association between thyroid hormones, thyroid antibodies and insulin resistance in euthyroid individuals: a population-based cohort. Diabetes Metab (2015) 41:480-8. doi:10.1016/j.diabet.2015.04.004

10. Hainer V, Zamrazilova H, Aldhoon Hainerova I. [Are the thyroid hormones and thyrotropin associated with cardiometabolic risks and insulin resistance even in euthyroid subjects?]. Vnitr Lek (2016) 62:63-7.

11. Kang D, Yin Q, Yan X, Song H, Gao G, Liang J, et al. Serum cholesterol levels in middle-aged euthyroid subjects with positive thyroid peroxidase antibodies. Int J Clin Exp Med (2015) 8:21623-8.

12. Kusminski CM, Bickel PE, Scherer PE. Targeting adipose tissue in the treatment of obesity-associated diabetes. Nat Rev Drug Discov (2016) 15:639-60. doi:10.1038/nrd.2016.75

13. Knudsen N, Laurberg P, Rasmussen LB, Bulow I, Perrild H, Ovesen L, et al. Small differences in thyroid function may be important for body mass index and the occurrence of obesity in the population. JClin Endocrinol Metab (2005) 90:4019-24. doi:10.1210/jc.2004-2225

14. Michalaki MA, Vagenakis AG, Leonardou AS, Argentou MN, Habeos IG, Makri MG, et al. Thyroid function in humans with morbid obesity. Thyroid (2006) 16:73-8. doi:10.1089/thy.2006.16.73

15. Kok P, Roelfsema F, Langendonk JG, Frolich M, Burggraaf J, Meinders AE, et al. High circulating thyrotropin levels in obese women are reduced after body weight loss induced by caloric restriction. J Clin Endocrinol Metab (2005) 90:4659-63. doi:10.1210/jc.2005-0920

16. Matthews DR, Hosker JP, Rudenski AS, Naylor BA, Treacher DF, Turner RC. Homeostasis model assessment: insulin resistance and beta-cell function from fasting plasma glucose and insulin concentrations in man. Diabetologia (1985) 28:412-9. doi:10.1007/BF00280883

17. Karanikas G, Schuetz M, Wahl K, Paul M, Kontur S, Pietschmann P, et al. Relation of anti-TPO autoantibody titre and T-lymphocyte cytokine production patterns in Hashimoto's thyroiditis. Clin Endocrinol (Oxf) (2005) 63:191-6. doi:10.1111/j.1365-2265.2005.02324.x

18. Park HJ, Kim J, Han EJ, Park SE, Park CY, Lee WY, et al. Association of low baseline free thyroxin levels with progression of coronary artery calcification over 4 years in euthyroid subjects: the Kangbuk Samsung Health Study. Clin Endocrinol (Oxf) (2016) 84:889-95. doi:10.1111/cen.12946

19. Chang X, Zhang B, Lihua L, Feng Z. T3 inhibits the calcification of vascular smooth muscle cells and the potential mechanism. Am J Transl Res (2016) 8:4694-704.

20. Dardano A, Ghiadoni L, Plantinga Y, Caraccio N, Bemi A, Duranti E, et al. Recombinant human thyrotropin reduces endothelium-dependent vasodilation in patients monitored for differentiated thyroid carcinoma. JClin Endocrinol Metab (2006) 91:4175-8. doi:10.1210/jc.2006-0440

21. Tian L, Zhang L, Liu J, Guo T, Gao C, Ni J. Effects of TSH on the function of human umbilical vein endothelial cells. J Mol Endocrinol (2014) 52:215-22. doi:10.1530/JME-13-0119

22. Auer J, Berent R, Weber T, Lassnig E, Eber B. Thyroid function is associated with presence and severity of coronary atherosclerosis. Clin Cardiol (2003) 26:569-73. doi:10.1002/clc.4960261205

23. Sieminska L, Wojciechowska C, Walczak K, Borowski A, Marek B, Nowak M, et al. Associations between metabolic syndrome, serum thyrotropin, and thyroid antibodies status in postmenopausal women, and the role of interleukin-6. Endokrynol Pol (2015) 66:394-403. doi:10.5603/EP.2015.0049

24. Sun L, Zhang X, Dai F, Shen J, Ren C, Zuo C, et al. Elevated interleukin-lbeta in peripheral blood mononuclear cells contributes to the pathogenesis of autoimmune thyroid diseases, especially of Hashimoto thyroiditis. Endocr Res (2016) 41:185-92. doi:10.3109/07435800.2015.1124439

25. Ozderya A, Aydin K, Temizkan S, Dogru Abbasoglu S, Vural P, Altuntas Y. High circulating levels of sICAM-1 and sVCAM-1 in the patients with Hashimoto's thyroiditis. Endocr Res (2017) 42:110-6. doi:10.1080/07435800. 2016.1201100

26. Wang G, Dai J, Mao J, Zeng X, Yang X, Wang X. Folic acid reverses hyperresponsiveness of LPS-induced chemokine secretion from monocytes in patients with hyperhomocysteinemia. Atherosclerosis (2005) 179:395-402. doi:10.1016/j.atherosclerosis.2004.10.033

27. Wang G, Wei J, Guan Y, Jin N, Mao J, Wang X. Peroxisome proliferatoractivated receptor-gamma agonist rosiglitazone reduces clinical inflammatory responses in type 2 diabetes with coronary artery disease after coronary angioplasty. Metabolism (2005) 54:590-7. doi:10.1016/j.metabol.2004.11.017

28. CzarnockaB,Janota-BzowskiM,McIntoshRS, AsgharMS, WatsonPF,KempEH, et al. Immunoglobulin G kappa antithyroid peroxidase antibodies in Hashimoto's thyroiditis: epitope-mapping analysis. J Clin Endocrinol Metab (1997) 82:2639-44. doi:10.1210/jcem.82.8.4124

29. Prummel MF, Wiersinga WM. Thyroid peroxidase autoantibodies in euthyroid subjects. Best Pract Res Clin Endocrinol Metab (2005) 19:1-15. doi:10.1016/j. beem.2004.11.003

30. Ehlers M, Jordan AL, Feldkamp J, Fritzen R, Quadbeck B, Haase M, et al. Antithyroperoxidase antibody levels $>500 \mathrm{IU} / \mathrm{ml}$ indicate a moderately increased risk for developing hypothyroidism in autoimmune thyroiditis. Horm Metab Res (2016) 48:623-9. doi:10.1055/s-0042-112815

31. Gao N, Zhang W, Zhang YZ, Yang Q, Chen SH. Carotid intima-media thickness in patients with subclinical hypothyroidism: a meta-analysis. Atherosclerosis (2013) 227:18-25. doi:10.1016/j.atherosclerosis.2012.10.070

32. Garin MC, Arnold AM, Lee JS, Tracy RP, Cappola AR. Subclinical hypothyroidism, weight change, and body composition in the elderly: the Cardiovascular Health Study. JClin Endocrinol Metab (2014) 99:1220-6. doi:10.1210/jc.2013-3591

33. Montagnani M, Chen H, Barr VA, Quon MJ. Insulin-stimulated activation of eNOS is independent of $\mathrm{Ca}^{2+}$ but requires phosphorylation by Akt at Ser(1179). J Biol Chem (2001) 276:30392-8. doi:10.1074/jbc.M103702200

34. Taniguchi CM, Emanuelli B, Kahn CR. Critical nodes in signalling pathways: insights into insulin action. Nat Rev Mol Cell Biol (2006) 7:85-96. doi:10.1038/ nrm1837

35. Ambrosi B, Masserini B, Iorio L, Delnevo A, Malavazos AE, Morricone L, et al. Relationship of thyroid function with body mass index and insulinresistance in euthyroid obese subjects. J Endocrinol Invest (2010) 33:640-3. doi:10.3275/6952

36. Bakker SJ, ter Maaten JC, Popp-Snijders C, Heine RJ, Gans RO. Triiodothyronine: a link between the insulin resistance syndrome and blood pressure? J Hypertens (1999) 17:1725-30. doi:10.1097/00004872-199917120-00009

37. Bakker SJ, ter Maaten JC, Popp-Snijders C, Slaets JP, Heine RJ, Gans RO. The relationship between thyrotropin and low density lipoprotein cholesterol is modified by insulin sensitivity in healthy euthyroid subjects. J Clin Endocrinol Metab (2001) 86:1206-11. doi:10.1210/jc.86.3.1206

38. Tagliaferri V, Romualdi D, Guido M, Mancini A, De Cicco S, Di Florio C, et al. The link between metabolic features and TSH levels in polycystic ovary syndrome is modulated by the body weight: an euglycaemic-hyperinsulinaemic clamp study. Eur J Endocrinol (2016) 175:433-41. doi:10.1530/EJE-16-0358

Conflict of Interest Statement: The authors declare that the research was conducted in the absence of any commercial or financial relationships that could be construed as a potential conflict of interest.

Copyright $\odot 2018$ Liu, Duan, Fu and Wang. This is an open-access article distributed under the terms of the Creative Commons Attribution License (CC BY). The use, distribution or reproduction in other forums is permitted, provided the original author(s) and the copyright owner are credited and that the original publication in this journal is cited, in accordance with accepted academic practice. No use, distribution or reproduction is permitted which does not comply with these terms. 